chances of establishment of species the seeds of which germinate slowly, and in the invasion by alien species of areas already occupied by other plants (especially when this is a consequence of the grazing, and other activities, of animals) the value to any species of this behaviour in the seedling stage would also seem clear. In this connexion it would be extremely interesting to know if a similar phenomenon is shown by the seedlings of any dicotyledonous plants.

Welsh Plant Breeding Station, H. G. Chippindare. Aberystwyth, Dec. 7.

\section{Zeeman Effect of a Forbidden Line.}

THE Zeeman effect is one of the most powerful methods of finding the causes of the occurrence of forbidden lines. The selection rules which govern it are quite different for ordinary lines, for quadripole lines, and for lines the appearance of which is due to external electric fields.

Quite recently the Zeeman effect of quadripole lines has been experimentally investigated. We have now been able to obtain the Zeeman effect of a line which shows the characteristic features of a line due to the random electric fields of the discharge, for example, the components with $\Delta m=0$ are observable in the transverse direction as $\sigma$ - and $\pi$-components.

The mercury line $6{ }^{3} P_{2}-7{ }^{3} P_{2} \lambda=3680$ A., which is forbidden by the selection rule for the azimuthal quantum number, was investigated in a magnetic field of about 12,000 gauss. As light source an are in vacuum was used.

If this line was due to quadripole radiation, its Zeeman pattern would have been of the type $\frac{(3) 6}{2}$, with all the components of equal intensity as shown

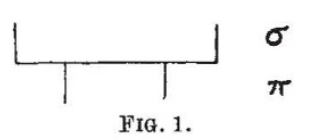

in Fig. 1. Assuming an electric field distributed at random, the type can be calculated by means of the Schrödinger perturbation theory (we are indebted to Dr. E. Majorana for this calculation), and one obtains the pattern of Fig. 2 of the type $\frac{(0)(3) 036}{2}$. (The numbers in the figure denote the calculated intensities of the components.) Moreover, we should expect that the line would be rather diffuse owing to the rapid variability of the electric fields, due to the ions which are present in the discharge and to the Stark effect shift.

What we actually observed without field was a rather diffuse line. In the magnetic field the line showed little alteration, but became a little broader, especially in $\pi$-polarisation.

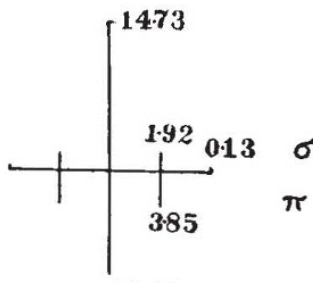

$11 \cdot 14$

FIG. 2.
The diffusion was real because the Zeeman pattern of other lines on the same plate, for example, $\lambda=3662.88$ $6{ }^{3} P_{2}-6{ }^{3} D_{1}$ of mercury, Zeeman pattern $\frac{(0)(2) 135}{2}$, were resolved. Anyhow, from our photographs we conclude that the Zeeman pattern was a centred one both in $\pi$-and $\sigma$-polarisation. If the Zeeman type had been as in Fig. 1, corresponding to quadripole radiation, we should undoubtedly have seen a non-centred type and probably should have resolved components separated by 3 and 6 normal units, even with a line originally so diffuse, if they had occurred.

Moreover, the broadening of the $\pi$-components is a confirmation of our interpretation, because, as shown in Fig. 2, the lateral components in $\pi$-polarisation are in the same position as, but double the intensity of, those in $\sigma$-polarisation.

We hope to repeat the experiment with a stronger magnetic field.

We are indebted to Prof. P. Zeeman for his interest and advice in connexion with this investigation.

E. SEgRì.

C. J. BAKKER.

Laboratory "Physica" of the University, Amsterdam.

\section{Neon-Helium Bands.}

IN the course of some experiments on the spectrum of the negative glow, some bands near $4000 \mathrm{~A}$. were observed in the negative glow of neon-helium mixtures. The definite experiments were carried out in the follow. ing way.

Two glow lamps (nickel plate cathode, ring anode) were joined by a glass tube; in the tube was a small glass bulb which separated the gases from another, and the bulb could be shattered by a small iron ball. One glow lamp was filled (after degassing) with neon and the other with helium, both at a pressure of $6 \mathrm{~mm}$. of mercury. Spectrograms were taken of the two negative glows with a small glass spectrograph, Fig. 1.

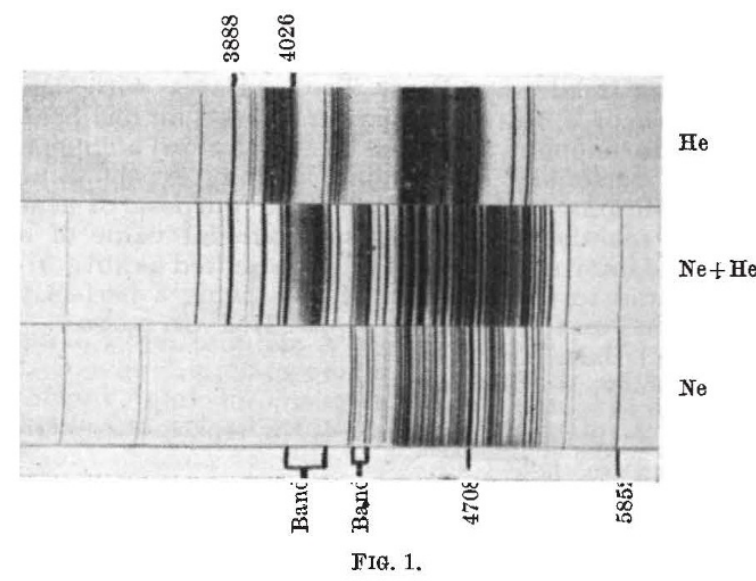

In the neon glow the neon arc- and spark-lines were observed, in the helium glow the helium lines and the $\mathrm{He}_{2}$-bands were present. Then the small glass bulb was shattered, so that the gases were mixed and a new spectrogram was taken. The $\mathrm{He}_{2}$-bands had now disappeared, but besides the neon and helium lines two bands were observed, the stronger one between the helium lines 4026 and 4121 , the other one between 4219 and 4276. Although the bands could not be resolved, it seems very improbable that they would coincide with $\mathrm{He}_{2}$-bands. The most probable explanation seems that the bands are to be attributed to a compound of neon and helium, such as Ne-He.

Prof. Coster in Groningen was so kind as to take some spectrograms of these bands with a grating ; on some plates the bands were partly resolved, but the intensity was too low to photograph the lines with greater dis. persion. To obtain a greater intensity I looked for these bands in a direct current positive column in a neon-helium mixture, but I did not obtain the bands. ${ }^{1}$ Maybe this can be attributed to the fact that in the negative glow a great velocity range of electrons is

No. 3243 , VoL. 128] 